\title{
Modeling the occurrence of unsafe behaviors based on dimensions of safety climate and organizational leadership style in oil platforms using fuzzy logic approach
}

\section{Mohsen Sadeghi Yarandi}

Department of Occupational Health Engineering, School of Public Health, Tehran University of Medical Sciences, Tehran, Iran https://orcid.org/0000-0002-2512-8591

\section{Ehsan Rastegarzadeh}

Department of Industrial Safety Engineering, Tabnak Lamerd Institute of Higher Education, Lamerd, Iran Ahmad Soltanzadeh

Department of Occupational Health Engineering, Faculty of Health, Qom University of Medical Sciences, Qom, Iran

Ali Karimi ( $\sim$ hse.expert2020@gmail.com )

Department of Occupational Health Engineering, School of Public Health, Tehran University of Medical Sciences, Tehran, Iran https://orcid.org/0000-0001-6924-2903

\section{Sevda Panahi}

Department of Occupational Health Engineering, School of Public Health, Tehran University of Medical Sciences, Tehran, Iran

\section{Research Article}

Keywords: Unsafe behavior, Leadership style, Safety climate, Fuzzy logarithmic model, Oil platform.

Posted Date: March 24th, 2021

DOl: https://doi.org/10.21203/rs.3.rs-337281/v1

License: (1) This work is licensed under a Creative Commons Attribution 4.0 International License. Read Full License 


\section{Abstract}

Background: Offshore oil and gas platforms have a high potential for major accidents due to sensitive operational conditions. Safety climate and leadership style have been identified as two key factors in the occurrence of unsafe behaviors. This study aimed to modeling the occurrence of unsafe behaviors based on dimensions of safety climate and organizational leadership style in oil platforms using fuzzy logic approach.

Methods: This cross-sectional and modeling study was conducted in 2019 among five oil platforms located in the Persian Gulf. The sample size was 291 employees. To measure organizational leadership style and safety climate, multifactor leadership and standard occupational safety climate questionnaires were used, respectively. To determine unsafe behaviors, a checklist of unsafe acts was used. Data analysis was performed using one-way ANOVA and Pearson correlation coefficients in SPSS version 25. Fuzzy logarithmic model and MATLAB software were used for modeling.

Results: There was a significant inverse relationship between transformational and transactional leadership style and the occurrence of unsafe behaviors. Moreover, finding revealed that there was a significant correlation between the dimensions of safety of workplace and the neglecting dangers and the occurrence of unsafe behaviors (P-value< 0.05 ). The results of the fuzzy logarithmic model also showed that mentioned parameters are important risk factors for predicting the occurrence of unsafe behaviors in oil platforms.

Conclusion: Findings revealed that transformational and transactional leadership styles, safety of workplace and neglecting dangers in oil platforms are among important factors in the occurrence of unsafe behaviors. Using fuzzy logarithmic models can also be an effective way to predict and control the occurrence of unsafe behaviors in oil platforms.

\section{Introduction}

Occupational accidents are one of the major problems in countries that impress different psychological, health, economic and organizational dimensions. According to available statistics, occupational accidents are the third leading cause of death in the world and the second leading cause of death in Iran. ${ }^{1}$ The International Labor Organization (ILO) reports that there are two million deaths annually due to accidents in workplaces. ${ }^{2}$ Studies show that the root cause of more than 80 percent of accidents is unsafe actions and the other 20 percent is due to unsafe conditions and other related factors. ${ }^{3}$ Behaviorbased safety identifies and modifies behaviors in the workplace that are effective in causing accidents and, to achieve this goal, considers the organizational and social infrastructures of these behaviors. ${ }^{4}$ Behavior is a reaction arising from an action or external action of the living being. This behavior in humans is more complex than other beings. The reason for this issue is the complexity of the human 
mental processes that make up the subject of psychological knowledge. Stability in behavior is very important. Because, based on that, one can predict the behavior. ${ }^{5}$ Research to identify the causes of accidents has shown that attitudes, behaviors, and cultures account for 85 to 98 percent of occupational accidents caused by unsafe behavior. ${ }^{6}$

Many organizational factors are used to determine safe and unsafe behaviors, the most prominent of which is the safety climate. ${ }^{7}$ Safety climate is one of the topics that has been introduced into the scope of safety and accident prevention in recent decades. Various definitions have been provided for the safety climate. According to the British Confederation of Industry, the safety climate is the opinions and beliefs of all organizations about an accident and illness. ${ }^{8}$ The safety climate examines employees' perceptions about the workplace, the level of interest in safety management and safety measures, and the extent of people's participation in risk control. ${ }^{9}$ Safety culture and consequently safety climate, like other psychosocial characteristics, have an inextricable link to behavior. ${ }^{10}$ Employees in an organization adjust their behaviors based on the level of understanding and analysis of organizational structures and interactions. The concept of safety climate emphasizes workers' perceptions. Safety climate as an important indicator determines the efficiency and safety performance of an organization and can predict the occurrence of occupational accidents. ${ }^{11,12}$

Another important and influential factor in the prevalence of unsafe behaviors is organizational leadership style. Leadership style is one of the organizational variables that relatively little research has been done on the scope of safety knowledge. Previous studies have shown that paying attention to leadership style in organizations and managers' commitment to safety-related issues can reduce the occurrence of unsafe behaviors and consequently reduce the accidents in the workplace. ${ }^{13,14}$ Few studies have been conducted on how the components of organizational leadership style can influence the creation of appropriate safety culture and climate to reduce the incidence of unsafe behaviors in the workplace. ${ }^{14}$ As stated above, the safety climate acts as a mediating parameter between organizational leadership style and the occurrence of unsafe behaviors, and appropriate leadership style in the field of safety can lead to create a favorable safety climate and consequently reduce the occurrence of unsafe behaviors and accidents in workplaces. ${ }^{15}$ Researchers have shown that positive attitudes toward safety, the manager's commitment to safety issues and the appropriate workplace safety climate have been effective in both accepting and maintaining occupational safety measures and reducing occupational accidents. ${ }^{16}$ The international labor organization estimates that more than 350,000 deaths and 330 million accidents occur each year in the workplace and their damage is estimated at $4 \%$ of global GDP. ${ }^{17}$ Given that Iran's population accounts for $1 \%$ of the world population, the number of occupational deaths and injuries can be estimated at 3,500 deaths and 3 million three hundred thousand injuries per year. ${ }^{3}$

In recent years, safety problems in oil platforms around the world have raised concerns. Working on these platforms involves a series of high-risk activities with high psychological and physical burden. ${ }^{18}$ Offshore oil and gas platforms have a high potential for major and critical accidents due to sensitive operational conditions, the existence of vital equipment and significant volumes of hydrocarbon materials. Previous 
studies have shown that one of the occupations with high volumes of unsafe behaviors and the risk of a variety of occupational accidents is working on oil platforms. ${ }^{19,20}$ Therefore, considering the importance of safety climate and leadership style as crucial issues in increasing safety levels and reducing accident rates in sensitive working environments such as offshore oil and gas platforms and their interaction as key elements in the emergence of unsafe behaviors in the workplace, this study aimed to modeling the occurrence of unsafe behaviors based on dimensions of safety climate and organizational leadership style in oil platforms using fuzzy logic approach.

\section{Methods}

\section{Study population}

This cross-sectional and modeling study was conducted in 2019 among five oil platforms located in the Persian Gulf in southern Iran. The statistical population consisted of 564 workers in five oil platforms. The sample size was determined by applying the Cochran formula with a margin of error of 0.04 equal to 291 employees. They were selected by systematic random sampling from the staff of five oil platforms. Finally, according to the response rate and elimination of incomplete and confusing questionnaires, 280 workers were studied (response rate was $96 \%$ ). Before conducting the study and completing the questionnaires and checklists, the necessary coordination was done and sufficient information was provided to the participants. Inclusion criteria were having at least one year of work experience and exclusion criteria were also defined as lack of enough consent to participate in the study. employees were allowed to withdraw from the study at any stage if they did not have consent.

\section{Data collection tools}

\section{Leadership style measurement}

Multifactor leadership questionnaire (MLQ) designed by Bass and Avolio in 1977 was used to measure leadership style. This questionnaire evaluates three different leadership styles: transformational, transactional (exchange), and passive-avoidant. It allows individuals to measure how they perceive themselves with regard to specific leadership behaviors, but the heart of the MLQ comes in the rater/other feedback that is enabled with the rater form. The MLQ was designed with the 360-degree feedback method. Participants are asked to respond to 45 items in the MLQ 5x-Short (the current, classic version) using a five-point behavioral scale ("Not at all" to "Frequently if not always"). ${ }^{21}$

\section{Safety climate measurement}

To measure safety climate, a standard questionnaire of occupational safety climate with 37 questions including 8 components (management commitment for safety and priority of safety matters ( 10 questions), the knowledge of the workers and following safety rules (7 questions), the attitude of the workers regarding safety (4 questions), cooperation of the workers and commitment to following safety (5 questions), safety of workplace (4 questions), priority of safety over products (2 questions), and 
neglecting dangers ( 2 questions) were used. Scoring was performed on a five-point Likert scale ( $1=$ strongly disagree, 2 = disagree, $3=$ no idea, $4=$ agree, 5 = strongly agree). The validity and reliability of this questionnaire have been confirmed in previous studies. ${ }^{22,23}$

\section{Matching variables measurement tools}

Since the number of factors in leadership style and safety climate questionnaires are different for various dimensions, and in order to uniformed expression and facilitate comparison of different dimensions of leadership style and safety climate in oil platforms, fuzzy scaling was used to eliminate the effect of the number of questions. The fuzzy scaling formula is as follows:

$$
\mathrm{Fi}=\frac{\sum_{\mathrm{j}}^{\mathrm{n}} \mathrm{Xij}_{\mathrm{j}}-(5 \times \mathrm{r})}{(5 \times \mathrm{r})-\mathrm{r}}
$$

Where $\mathrm{Fi}$ is a questionnaire rating for answering the number $\mathrm{i}$, which ranges from zero to one, $\mathrm{ij}$ is the score of the question $\mathrm{j}$ according to Likert scale, $r$ is the number of items and $n$ is equal to the sample size (number of respondents). For example, in the safety climate questionnaire, the dimension of management commitment for safety and priority of safety matters has 10 questions and the neglecting dangers dimension has 2 questions. Given that the sum of the scores for each dimension is calculated on a Likert basis, naturally, the dimension with the fewer questions receives the lower percentage of points compared to the part with the more questions. So by fuzzy scaling, the effect of the number of questions omitted and the score of each dimension will be calculated from zero to 100 .

\section{Unsafe behaviors measurement}

Direct observation was used to record unsafe behaviors while workers performing the task. For this purpose, a checklist of unsafe behaviors was prepared based on the risk factors of accidents and nearmiss that have taken place on the oil platforms over the past 10 years and then approved by experts and university professors to gain the capability for using as a tool for measuring unsafe behaviors. The checklist consisted of 15 questions and was completed by 10 certified safety experts who worked on five oil platforms. In this step, each worker was being monitored in an intangible way for 30 to 45 minutes to record their unsafe behaviors during working time. The range of unsafe behavior score was between 0 and $100 \%$.

\section{Data analysis}

In the present study, descriptive statistics such as mean, standard deviation and frequency were presented. Kolmogorov-Smirnov statistical test was used to evaluate the normality/non-normality of the data distribution. By confirming the normality of the data distribution (P-value> 0.05$)$, data were analyzed using One-way analysis of variance (ANOVA) and Pearson's correlation coefficient. All tests were performed at the significant level of 0.05 and the data were analyzed by SPSS software version 25 . 


\section{Design of fuzzy logarithmic model}

In this study, based on research literature and fuzzy sets, fuzzy inference system was designed to evaluate safety performance based on organizational factors. In the present study, the triangular function was used for fuzzy modeling. The fuzzy inference method was used by the Mamdani method. All mathematical operations were performed in MATLAB software (version 2018a). After model development and assurance of model accuracy, the developed system was used for a case study to evaluate safety performance. Initially, input and output variables were determined. According to the calculations performed in the previous steps in SPSS software, only the components that had a significant relationship with the occurrence of unsafe behaviors and were statistically significant (accounted for the highest frequency) were considered as input variables in MATLAB software. After obtaining the statistical information, the dominant dimensions of organizational leadership style and occupational safety climate were considered as system inputs and unsafe behaviors were identified as the only dependent variable and system output. Figure 1 shows a schematic view of how the fuzzy inference system works in this study.

The fuzzy logic system is actually the path and rules for studying the output variable changes according to input variable changes. This logic expresses the degree of membership of each variable in a set.

Formula 1 illustrates how to investigate the triangular variable in fuzzy logic.

$$
\mu_{x}(x)=\left\{\begin{array}{cc}
\left(x-a^{l}\right) /\left(a^{m}-a^{l}\right), & a^{l} \leq x<a^{m} \\
1, & x=a^{m} \\
\left(a^{r}-x\right) /\left(a^{r}-a^{m}\right), & a^{m}<x \leq a^{r} \\
0, & \text { otherwise }
\end{array}\right. \text { Formula 1 }
$$

Figure 2 also shows an image of a triangular function.

\section{Fuzzification of input and output variables}

Fuzzification is a step to determine the degree to which an input data belongs to each of the appropriate fuzzy sets via the membership functions. Using the data collected from the statistical sample of research in each dimensions of leadership style, safety climate and unsafe behaviors, significant components of leadership style (transformational and transactional leadership) and safety climate (neglecting danger and safety of workplace) were considered as input variables. Each of the variables was placed in fuzzy intervals with appropriate distances.

The inference rules (fuzzy inference system) were formulated considering four input variables, each of variables is divided into three linguistic variables. In an ideal condition 81 (four $3 \times 3$ matrices) law can be explained. After preparation, the fuzzy inference system was approved by academic professors and experts and then entered into the inference system. 


\section{Results}

The majority of participants were in the age group of 31 to 40 years $(61.8 \%), 56.4 \%$ were formal in terms of type of employment contract, and the majority of participants had a bachelor's degree (54.3\%). Finally, it was found that $91.1 \%$ of all studied workers did not experience any accidents in the last year. Investigation of the participant's distribution in the five oil platforms showed that $33.21,30,12.14,17.86$ and $6.79 \%$ of the workers are working in A, B, C, D and E oil platforms, respectively. Other obtained demographic data are presented in Table 2

\section{The occurrence of unsafe behaviors}

Frequently distribution of unsafe behaviors showed that 162 workers did not show any unsafe behaviors and were so-called safe and 10 workers had completely unsafe behaviors. Other results of the study of unsafe behaviors in the present study are presented in Table 3.

Based on the results of Table 4, the most unsafe behaviors were related to $D$ and $E$ platforms. Overall, the average estimated unsafe behavior among all studied workers was $8 \%$.

One-way analysis of variance (ANOVA) showed that there was a significant relationship between the mean percentage of unsafe behaviors in different platforms $(P$-value $=0.004)$.

\section{Organizational leadership style}

The leadership style estimation, which ranges from zero to $100 \%$, is presented in Table 5 . Based on the obtained results, the transformational leadership style in studied platforms is dominant over the other two leadership styles.

The estimation of the leadership style dimensions is shown in Table 6. The leadership style components have a score range between zero and $100 \%$ like the other variables in the study, which means that each leadership style dimension has a score closer to $100 \%$, is fully institutionalized and $100 \%$ relevant to the platform. Based on the results in Table 6, the highest scores were obtained in the two components of intellectual stimulation and contingent reward and then individualized consideration.

Investigating the relationship between different leadership styles and the occurrence of unsafe behaviors using Pearson correlation coefficient revealed that the values of correlation coefficients between transformational and transactional leadership style and the occurrence of unsafe behaviors were -0.138 and -0.191 , respectively and there was a significant inverse relationship between these two items ( $P$-value $<0.05)$.

\section{Occupational safety climate}

The mean score of safety climate among all studied subjects was $136.71 \pm 10.94$. In order to compare the values of the overall score of the safety climate among the oil platforms, all values were matched in the range of 0-100 percent and are presented in Table 7. According to the results, the highest average 
safety climate was found in $\mathrm{C}$ and $\mathrm{D}$ platforms and the average safety climate among all platforms was $67 \%$.

The results of one-way ANOVA test showed that there was no significant relationship between the mean score of safety climate in different oil platforms (P-value $=0.772)$. It was also found that there was a significant relationship between mean values of different dimensions of safety climate in different platforms (P-value $=0.04$ ). Based on the results of Table 8 , the highest score was found in the dimension of attitude of the workers regarding safety that indicating the mentioned dimension was completely institutionalized among the studied workers.

Investigation of the relationship between safety climate and its different dimensions with unsafe behavior showed that there was no statistically significant relationship between knowledge of the workers and following safety rules, attitude of the workers regarding safety and cooperation of the workers and commitment to following safety dimensions with occurrence of unsafe behavior (P-value $>0.05$ ). Pearson's correlation coefficient between safety of workplace and unsafe behavior was -0.185 and there was a significant relationship between the mentioned parameters.

There was also a significant relationship between the score of neglecting dangers dimension and unsafe behavior ( $P$-value $<0.05$ ). Other values of correlation between dimensions of safety climate and unsafe behavior are presented in Table 9. Analysis of the relationship between demographic variables and unsafe behavior using one-way analysis of variance revealed that there is a significant relationship between education level and occurrence of unsafe behavior ( $P$-value $<0.05)$. The relationship between other demographic parameters and unsafe behavior was not significant. It was also found that there was a significant relationship between experience of accident and near-miss in workplace and occurrence of unsafe behaviors (P-value <0.05).

\section{Modeling unsafe behavior}

In Figure 3, the relationship between transformational and transactional leadership styles with the occurrence of unsafe behaviors is shown in 3D.

In Figure 4 the relationship between unsafe behaviors with each of these leadership styles is presented in 2D.

The relationship between the dimensions of neglecting dangers and safety of workplace with the occurrence of unsafe behaviors is also shown in Fig. 5 in 3D.

Also, the relationship between unsafe behavior with two mentioned dimension is presented in Fig. 6 in 2D.

\section{Discussion}

In the present study, the influence of risk factors such as organizational leadership style and safety climate and their different dimensions as an important factor in the development of unsafe behaviors 
using fuzzy logarithmic models in five oil platforms was studied for the first time in Iran. Investigating the relationship between different leadership styles and the occurrence of unsafe behaviors using Pearson correlation coefficient revealed that the values of correlation coefficients between transformational and transactional leadership style and the occurrence of unsafe behaviors were -0.138 and -0.191 , respectively and there was a significant inverse relationship between these two items ( $P$-value $<0.05)$. Previous studies have shown that organizational leadership style is an effective factor in the level of organization safety and the occurrence of unsafe behaviors and transformational leadership can predict occupational accidents and the occurrence of unsafe acts through effects on safety climate. ${ }^{24,25}$ Fernández-Muñiz et al reported in their study that leadership factor is an influential parameter in the behavior and safety performance of individuals in the process industries, which is consistent with the results of the present study. ${ }^{14}$ The finding of the study conducted by Tsung-Chih Wu et al. revealed that organizational leadership can improve the safety climate and consequently safety performance of workers in an organization by developing some strategies. ${ }^{15}$

The highest average of safety climate was found in $C$ and $D$ platforms and the average safety climate for all platforms was $67 \%$ (Table 7). It was found that there is a significant relationship between mean values of different dimensions of safety climate in different platforms. Among the eight dimensions of safety climate, neglecting dangers as the weakest dimension with the mean score of $42.86 \%$ and the attitude of the workers regarding safety with the mean score of $93.48 \%$ was the strongest dimension of research (Table 8). This indicates that the dimension of knowledge and attitude of employees towards safety issues is completely institutionalized by the training program that was provided on the studied platforms. The lowest average safety climate was found in E oil platform (67\%), and the results of the survey of unsafe behaviors in different platforms revealed that the most frequent unsafe acts were in this oil platform (16\%). This reflects the impact of the safety climate and its different dimensions on the occurrence of unsafe behavior. A study conducted by Rahimi et al. in a petrochemical industry showed that safety climate is one of the most important factors affecting unsafe behaviors in different working environments. ${ }^{26}$ The results of the study by Yu et al. among Chinese miners also showed that safety climate is an effective parameter in reducing the incidence of unsafe behaviors and a favorable safety climate in workplaces can also reduce job stress and burnout. ${ }^{27}$ Previous studies have shown that workers in oil platforms are exposed to high levels of physical and psychological risk factors, which can lead to many accidents in these working environments. ${ }^{18}$

Investigation of the relationship between safety climate and its different dimensions with unsafe behavior showed that there was no statistically significant relationship between knowledge of the workers and following safety rules, attitude of the workers regarding safety and cooperation of the workers and commitment to following safety dimensions with occurrence of unsafe acts. This means that these three components of the completed questionnaires had no effect on unsafe behavior or had a slight effect. Given that the attitudes and safety knowledge of workers are high and desirable; it can be predicted that the occurrence of unsafe behaviors in the present study may be due to other risk factors. Pearson's correlation coefficient between workplace safety and unsafe behavior was - 0.185 and there 
was a significant inverse relationship between the two mentioned parameters. In other words, increasing workplace safety can ultimately reduce the incidence of unsafe behaviors. It was also found that there was a significant relationship between the dimension of neglecting dangers and the occurrence of unsafe behavior (Table 9). This indicates that as workers are more sensitive to the hazards surrounding them, the amount of unsafe behaviors decreases in the present study and vice versa. The finding of the study conducted by Oah et al. also showed that safety climate and safety leadership are among the most important parameters that influence employees' perception of risk concept and occurrence of unsafe behaviors that ultimately lead to reduction of accidents in working environments. ${ }^{11}$ Therefore, focusing on reducing unsafe behaviors in the workplace is the main option to increase the safety levels of different workplaces and consequently reduce the occurrence of occupational accidents.

In the present study, a fuzzy logarithmic model was used to accurately determine the role of transformational and transactional leadership style as well as the dimensions of neglecting danger and safety of workplace in predicting occurrence of unsafe behaviors. The relationship between the transactional (exchange) leadership style and unsafe behavior in the fuzzy model showed that in the approximate range of 0.65 to 0.95 the least risk of unsafe behavior was observed, which means that we can only experience a $15 \%$ increase in transactional leadership in this style. We will then experience up to $93 \%$ as a straight line with no change in the output of unsafe behavior (figure 3 ). So to improve this type of leadership style based on figures 3 and 4 we will get from $43.4 \%$ occurrence of unsafe behavior to $9.4 \%$, which means that by increasing and improving only $15 \%$ of the components of transactional leadership style, the amount of unsafe behavior occurrence will have decreased by $33.9 \%$. The effect of the transactional leadership style appears to be less than the transformational leadership style in this modeling so that we can increase or decrease up to $30 \%$ of the components of the transactional leadership style and not reach the critical point. So, according to figures 3 and 4 , we find that the components of a transformational leadership style will be more productive and work with them would be logical and optimal. Because, according to the model, if we move $20 \%$ toward transformational leadership and improve the components that create it, we will minimize the likelihood of unsafe behaviors occurrence.

The results of the study showed that the neglecting danger dimension had a score of 0.42 , meaning that studied workers accepted and ignored $42 \%$ of the risks on the platforms. In this sector, if the risk is neglected by another $14 \%$, there will be no significant impact on the amounts of unsafe behaviors, but it will fluctuate slightly, and these conditions will gradually increase after a $40 \%$ increment in the neglecting danger item. It was also found that the safety of workplace dimension had a score of 0.85 , the upper limit of which would be meaningless in the graph. Therefore, due to the high safety levels, the existing platform conditions are considered very good and this situation will remain acceptable if reduced by even $50 \%$ and without incremental changes in the occurrence of unsafe behavior. In other words, the safety coefficients of the studied platforms are considered to be $150 \%$ or one and a half times the standard (Figures 5 and 6). Finally, the obtained models during the present study revealed that unsafe behaviors 
can be effectively predicted using different dimensions of organizational leadership style as well as safety climate.

Offshore oil and gas platforms have a high potential for major events due to sensitive operating conditions, the existence of critical equipment, and significant volumes of hydrocarbons. Human behavior is very important in the occurrence of occupational accidents. This has led safety professionals to adopt an approach to preventing accidents that focuses on changing human behavior as the most important cause of workplace accident. The finding of this study showed that the organizational leadership style and safety climate in oil platforms are very important and effective factors in the occurrence of unsafe behaviors. Therefore, trying to develop a good leadership style and safety climate can be an effective step in reducing the occurrence of unsafe behaviors and consequently decreasing the occupational accidents on the oil platforms of Iran as one of the important and vital elements in the oil and gas industry. It was also found that using fuzzy models can be a useful tool for predicting and controlling unsafe behaviors in oil platforms. One of the limitations of the present study is the investigation of a limited number of oil platforms due to the time constraints and the long distance between the oil platforms considering the large area of the Persian Gulf. Therefore, considering the importance of working on these platforms and the high rate of accidents, it is recommended that future researchers investigate the occurrence of unsafe behaviors in other oil platforms with larger sample size and considering the role of other organizational risk factors as a predictor of unsafe behaviors in the workplace.

\section{Conclusion}

The results of this study showed that transformational and transactional leadership style, safety of workplace and neglecting dangers dimensions in oil platforms are among important and effective factors in the occurrence of unsafe behaviors. Using fuzzy logarithmic models can also be an effective way to predict and control the occurrence of unsafe behaviors in oil platforms.

\section{Declarations}

\section{Acknowledgments}

This article is the result of a master thesis of second author. The authors would like to express their special thanks to Tabnak Lamerd Institute of Higher Education for financial support.

Competing interests: None declared.

Ethical approval: This article supported by Tabnak Lamerd Institute of Higher Education. The study was approved by the ethics committee of Tabnak Lamerd Institute of Higher Education

\section{References}


1. Azadeh A, Nouri J, Fam IM. The impacts of macroergonomics on environmental protection and human performance in power plants. Journal of Environmental Health Science \& Engineering. 2005;2(1):60-6.

2. Soltanzadeh A, Mohammadfam I, Mahmoudi S, Savareh BA, Arani AM. Analysis and forecasting the severity of construction accidents using artificial neural network. Safety Promotion and Injury Prevention. 2017;4(3):185-92.

3. Shappell SA, Wiegmann DA. The human factors analysis and classification system--HFACS. 2000.

4. DeJoy DM. Behavior change versus culture change: Divergent approaches to managing workplace safety. Safety science. 2005;43(2):105-29.

5. Robbins SP, Judge T. Essentials of organizational behavior: Prentice Hall Upper Saddle River, NJ; 2003.

6. Dilley H, Kleiner BH. Creating a culture of safety. Work study. 1996.

7. Buck MA. Proactive personality and big five traits in supervisors and workgroup members: Effects on safety climate and safety motivation. 2011.

8. Heidari M, Farshad A, Arghami S. Astudy on relationship between production link worker's safety attitude and their safe act in of arak metal industry. Iran Occupational Health. 2007;4(3):1-9.

9. Zohar D. The effects of leadership dimensions, safety climate, and assigned priorities on minor injuries in work groups. Journal of organizational behavior. 2002;23(1):75-92.

10. Myers DJ, Nyce JM, Dekker SW. Setting culture apart: Distinguishing culture from behavior and social structure in safety and injury research. Accident Analysis \& Prevention. 2014;68:25-9.

11. Oah S, Na R, Moon K. The influence of safety climate, safety leadership, workload, and accident experiences on risk Perception: a study of Korean manufacturing workers. Safety and health at work. 2018;9(4):427-33.

12. Chen $Y, M c C a b e B$, Hyatt $D$. A resilience safety climate model predicting construction safety performance. Safety science. 2018;109:434-45.

13. Inness M, Turner N, Barling J, Stride CB. Transformational leadership and employee safety performance: A within-person, between-jobs design. Journal of Occupational Health Psychology. 2010;15(3):279.

14. Fernández-Muñiz B, Montes-Peón JM, Vázquez-Ordás CJ. The role of safety leadership and working conditions in safety performance in process industries. Journal of Loss Prevention in the Process Industries. 2017;50:403-15.

15. Wu T-C, Chen C-H, Li C-C. A correlation among safety leadership, safety climate and safety performance. Journal of loss prevention in the process industries. 2008;21(3):307-18.

16. Shao T, Cao D-Z, Li H-Z, Kong J, Xia Z-L. Investigation on the association of safety perception and safety behaviors with occupational injuries in steel-workers. Chinese journal of industrial hygiene and occupational diseases. 2004;22(6):416-8. 
17. Hämäläinen P, Takala J, Saarela KL. Global estimates of occupational accidents. Safety science. 2006;44(2):137-56.

18. Sun Z-Y, Zhou J-L, Gan L-F. Safety assessment in oil drilling work system based on empirical study and Analytic Network Process. Safety science. 2018;105:86-97.

19. Zhou $A$, Wang $K$, Zhang $H$. Human factor risk control for oil and gas drilling industry. Journal of Petroleum Science and Engineering. 2017;159:581-7.

20. Quanmin Z, Hong Z, Jianchun F. Human factor risk quantification for oil and gas drilling operation. Procedia Engineering. 2011;18:312-7.

21. Avolio BJ, Bass BM. Multifactor leadership questionnaire (MLQ). Mind Garden. 2004;29.

22. Zeydi IM, Heydarnia A, Niknami S. Safety climate measurement at workplace: Development, validity and reliability. Payesh (Health Monitor). 2011;10(2):157-65.

23. Yari S, Naseri MH, Akbari H, Shahsavari S, Akbari H. Interaction of Safety Climate and Safety Culture: A Model for Cancer Treatment Centers. Asian Pacific journal of cancer prevention: APJCP. 2019;20(3):961.

24. Pordanjani TR, Ebrahimi AM. The role of transformational leadership style and work-safety tension in prediction of incident reporting rate. Safety Promotion and Injury Prevention. 2017;4(4):231-8.

25. Barling J, Loughlin C, Kelloway EK. Development and test of a model linking safety-specific transformational leadership and occupational safety. Journal of applied psychology. 2002;87(3):488.

26. Rahimi Pordanjani T, Mohamadzade Ebrahimi A, Mehdizade H, Khoshniat M. A study of relationship between dimensions of safety climate with safe behaviors in the petrochemical industry. OCCUPATIONAL MEDICINE Quarterly Journal. 2015;6(4):1-11.

27. Yu M, Li J. Psychosocial safety climate and unsafe behavior among miners in China: the mediating role of work stress and job burnout. Psychology, health \& medicine. 2019:1-9.

\section{Tables}

Table1. Fuzzy numbers of variables

\begin{tabular}{|c|c|c|c|c|c|}
\hline \multirow{2}{*}{$\begin{array}{l}\text { Terbal } \\
\text { riables }\end{array}$} & \multicolumn{2}{|c|}{ Leadership style } & \multicolumn{2}{|c|}{ Safety climate } & \multirow{2}{*}{$\begin{array}{c}\text { Unsafe } \\
\text { behaviors }\end{array}$} \\
\hline & Transformational & Transactional & $\begin{array}{c}\text { Neglecting } \\
\text { danger }\end{array}$ & $\begin{array}{c}\text { Safety of } \\
\text { workplace }\end{array}$ & \\
\hline Low & {$\left[\begin{array}{lll}0 & 0.38 & 0.58\end{array}\right]$} & {$\left[\begin{array}{lll}0 & 0.41 & 0.53\end{array}\right]$} & {$\left[\begin{array}{llll}0 & 0.25 & 0.5\end{array}\right]$} & {$\left[\begin{array}{llll}0 & 0.25 & 0.5\end{array}\right]$} & $\begin{array}{r}0.08 \\
{\left[\begin{array}{ll}0 & 0.04\end{array}\right]}\end{array}$ \\
\hline oderate & {$\left[\begin{array}{lll}0.38 & 0.58 & 0.78\end{array}\right]$} & $\begin{array}{c}0.5] \\
{[0.41 \quad 0.65}\end{array}$ & {$\left[\begin{array}{llll}0.25 & 0.5 & 0.75\end{array}\right]$} & {$\left[\begin{array}{llll}0.25 & 0.5 & 0.75\end{array}\right]$} & $\begin{array}{c}0.08] \\
0.040 .16 \\
{[}\end{array}$ \\
\hline High & {$\left[\begin{array}{lll}0.58 & 0.78 & 1\end{array}\right]$} & {$\left[\begin{array}{lll}0.5 & 0.65 & 1\end{array}\right]$} & {$\left[\begin{array}{lll}0.5 & 0.75 & 1\end{array}\right]$} & {$\left[\begin{array}{lll}0.5 & 0.75 & 1\end{array}\right]$} & $\begin{array}{c}1] \\
0.080 .16 \\
{[}\end{array}$ \\
\hline
\end{tabular}


Table 2. Demographic information of the subjects (280 worker)

\begin{tabular}{|c|c|c|c|}
\hline \multicolumn{2}{|c|}{ Variable } & Frequency & Frequency (\%) \\
\hline \multirow{4}{*}{ Age } & Less than 30 years & 41 & 14.6 \\
\cline { 2 - 4 } & $40-31$ years & 173 & 61.8 \\
\cline { 2 - 4 } & $50-41$ years & 47 & 16.8 \\
\cline { 2 - 4 } & More than 51 years & 19 & 6.8 \\
\hline \multirow{4}{*}{ Contract type } & Official & 158 & 56.4 \\
\cline { 2 - 4 } & Contractual & 22 & 7.9 \\
\cline { 2 - 4 } & Treaty & 100 & 35.7 \\
\hline \multirow{4}{*}{ Education level } & Diploma & 74 & 26.4 \\
\cline { 2 - 4 } & Associate & 18 & 6.4 \\
\cline { 2 - 4 } & Bachelors & 152 & 54.3 \\
\cline { 2 - 4 } & Masters & 35 & 12.5 \\
\cline { 2 - 4 } History of the accident in the last year & P.H.D & 1 & 0.4 \\
\cline { 2 - 4 } & No Accident & 255 & 91.1 \\
\cline { 2 - 4 } & Accident & 4 & 1.4 \\
\cline { 2 - 4 } & Accident and near miss & 3 & 6.4 \\
\hline
\end{tabular}

Table 3. Frequency of unsafe behaviors among studied subjects

\begin{tabular}{c|c|c|c|}
\hline Type of behavior & Range of unsafe behavior changes & Frequency & Frequency (\%) \\
\hline Safe & No unsafe behavior & 162 & 57.8 \\
\hline A little unsafe & From 1 to 15 percent & 68 & 24.2 \\
\hline Almost unsafe & From 16 to 30 percent & 27 & 9.7 \\
\hline Very unsafe & From 31 to 45 percent & 13 & 4.7 \\
\hline Totally unsafe & From 46 to 100 percent & 10 & 3.6 \\
\hline
\end{tabular}

Table 4. Average percentage of unsafe behaviors according to oil platforms

\begin{tabular}{|c|c|c|c|c|}
\hline Platform & Average (\%) & Standard deviation (\%) & Minimum (\%) & Maximum (\%) \\
\hline A & 7 & 11 & 0 & 47 \\
\hline B & 6 & 9 & 0 & 47 \\
\hline C & 7 & 12 & 0 & 47 \\
\hline D & 12 & 20 & 0 & 73 \\
\hline E & 16 & 17 & 0 & 60 \\
\hline Total platforms & 8 & 13 & 0 & 73 \\
\hline
\end{tabular}




\begin{tabular}{|c|c|c|c|c|}
\hline Leadership style & Average (\%) & Standard deviation (\%) & Minimum (\%) & Maximum (\%) \\
\hline Transformational & 58.38 & 19.94 & 3.75 & 95.00 \\
\hline Transactional & 53.28 & 11.61 & 10.42 & 87.50 \\
\hline Passive-avoidant & 30.29 & 19.98 & 0.00 & 75.00 \\
\hline
\end{tabular}

Table 6. Leadership style dimensions' values

\begin{tabular}{|c|c|c|c|c|}
\hline Dimensions & $\begin{array}{c}\text { Average } \\
(\%)\end{array}$ & $\begin{array}{c}\text { Standard deviation } \\
\text { (\%) }\end{array}$ & $\begin{array}{l}\text { Minimum } \\
\text { (\%) }\end{array}$ & $\begin{array}{l}\text { Maximum } \\
(\%)\end{array}$ \\
\hline $\begin{array}{c}\text { Idealized } \\
\text { Ifluence (Attributes) }\end{array}$ & 55.38 & 23.98 & 0.00 & 100.00 \\
\hline $\begin{array}{c}\text { Idealized } \\
\text { Ifluence (Behaviors) }\end{array}$ & 57.05 & 22.04 & 0.00 & 100.00 \\
\hline pirational Motivation & 57.01 & 26.06 & 0.00 & 100.00 \\
\hline :ellectual Stimulation & 63.44 & 21.02 & 6.25 & 100.00 \\
\hline idualized Consideration & 59.02 & 23.50 & 0.00 & 100.00 \\
\hline Jontingent Reward & 60.67 & 21.19 & 0.00 & 100.00 \\
\hline $\begin{array}{l}\text { agement by Exception: } \\
\text { Active }\end{array}$ & 58.95 & 19.27 & 6.25 & 100.00 \\
\hline $\begin{array}{l}\text { agement by Exception: } \\
\text { Passive } \\
\end{array}$ & 40.22 & 16.88 & 0.00 & 87.50 \\
\hline Laissez-Faire & 30.29 & 19.98 & 0.00 & 75.00 \\
\hline
\end{tabular}

Table 7. Safety climate values based on studied platforms

\begin{tabular}{|c|c|c|c|c|}
\hline Platform & Average (\%) & Standard deviation (\%) & Minimum (\%) & Maximum (\%) \\
\hline A & 67 & 7 & 53 & 83 \\
\hline B & 67 & 5 & 58 & 84 \\
\hline C & 68 & 9 & 45 & 78 \\
\hline D & 68 & 6 & 53 & 81 \\
\hline E & 66 & 5 & 58 & 74 \\
\hline Total & 67 & 7 & 45 & 84 \\
\hline
\end{tabular}

Table 8. The values of different dimensions of safety climate among studied subjects 


\begin{tabular}{|c|c|c|c|c|}
\hline Dimensions & $\begin{array}{c}\text { Average } \\
(\%)\end{array}$ & $\begin{array}{c}\text { Standard } \\
\text { deviation (\%) }\end{array}$ & $\begin{array}{c}\text { Minimum } \\
(\%)\end{array}$ & $\begin{array}{c}\text { Maximum } \\
(\%)\end{array}$ \\
\hline $\begin{array}{c}\text { 1agement commitment for safety and } \\
\text { priority of safety matters }\end{array}$ & 57.46 & 17.41 & 15.00 & 95.00 \\
\hline $\begin{array}{c}\text { wledge of the workers and following } \\
\text { safety rules }\end{array}$ & 75.60 & 13.47 & 25.00 & 100.00 \\
\hline $\begin{array}{c}\text { tude of the workers regarding safety } \\
\text { Cooperation of the workers and } \\
\text { commitment to following safety }\end{array}$ & 93.48 & 12.12 & 0.00 & 100.00 \\
\hline Safety of workplace & 85.46 & 15.22 & 35.00 & 100.00 \\
\hline Emergency preparedness at work & 43.79 & 19.85 & 0.00 & 100.00 \\
\hline Priority of safety over products & 48.17 & 29.53 & 0.00 & 100.00 \\
\hline \multicolumn{1}{c|}{ Neglecting dangers } & 42.86 & 25.03 & 0.00 & 100.00 \\
\hline
\end{tabular}

Table 9. Pearson correlation coefficients between unsafe behavior and safety climate dimensions

\begin{tabular}{|c|c|c|c|c|c|}
\hline Dimensions & Variable & Value & Dimensions & Variable & Value \\
\hline \multirow[t]{2}{*}{$\begin{array}{l}\text { Management } \\
\text { commitment }\end{array}$} & $\begin{array}{c}\text { Correlation } \\
\text { coefficient }\end{array}$ & -0.112 & \multirow[t]{2}{*}{$\begin{array}{l}\text { Safety of } \\
\text { workplace }\end{array}$} & $\begin{array}{l}\text { Correlation } \\
\text { coefficient }\end{array}$ & -0.185 \\
\hline & P-value & 0.3 & & P-value & $0.002^{*}$ \\
\hline \multirow[t]{2}{*}{$\begin{array}{c}\text { Knowledge of the } \\
\text { workers }\end{array}$} & $\begin{array}{l}\text { Correlation } \\
\text { coefficient }\end{array}$ & 0.062 & \multirow[t]{2}{*}{$\begin{array}{c}\text { Emergency } \\
\text { preparedness }\end{array}$} & $\begin{array}{l}\text { Correlation } \\
\text { coefficient }\end{array}$ & 0.015 \\
\hline & $\begin{array}{l}\text { P-value } \\
\text { Pell }\end{array}$ & 0.301 & & P-value & 0.798 \\
\hline \multirow[t]{2}{*}{$\begin{array}{l}\text { Attitude of the } \\
\text { workers }\end{array}$} & $\begin{array}{c}\text { Correlation } \\
\text { coefficient }\end{array}$ & 0.098 & \multirow[t]{2}{*}{ Priority of safety } & $\begin{array}{c}\text { Correlation } \\
\text { coefficient }\end{array}$ & 0.048 \\
\hline & $\begin{array}{l}\text { P-value } \\
\text { P- }\end{array}$ & 0.103 & & P-value & 0.428 \\
\hline \multirow[t]{2}{*}{$\begin{array}{c}\text { Cooperation of the } \\
\text { workers }\end{array}$} & $\begin{array}{c}\text { Correlation } \\
\text { coefficient }\end{array}$ & 0.027 & \multirow[t]{2}{*}{$\begin{array}{c}\text { Neglecting } \\
\text { dangers }\end{array}$} & $\begin{array}{c}\text { Correlation } \\
\text { coefficient }\end{array}$ & 0.148 \\
\hline & P-value & 0.651 & & P-value & $0.013^{*}$ \\
\hline
\end{tabular}

* Significant correlation (P-value <0.05).

\section{Figures}




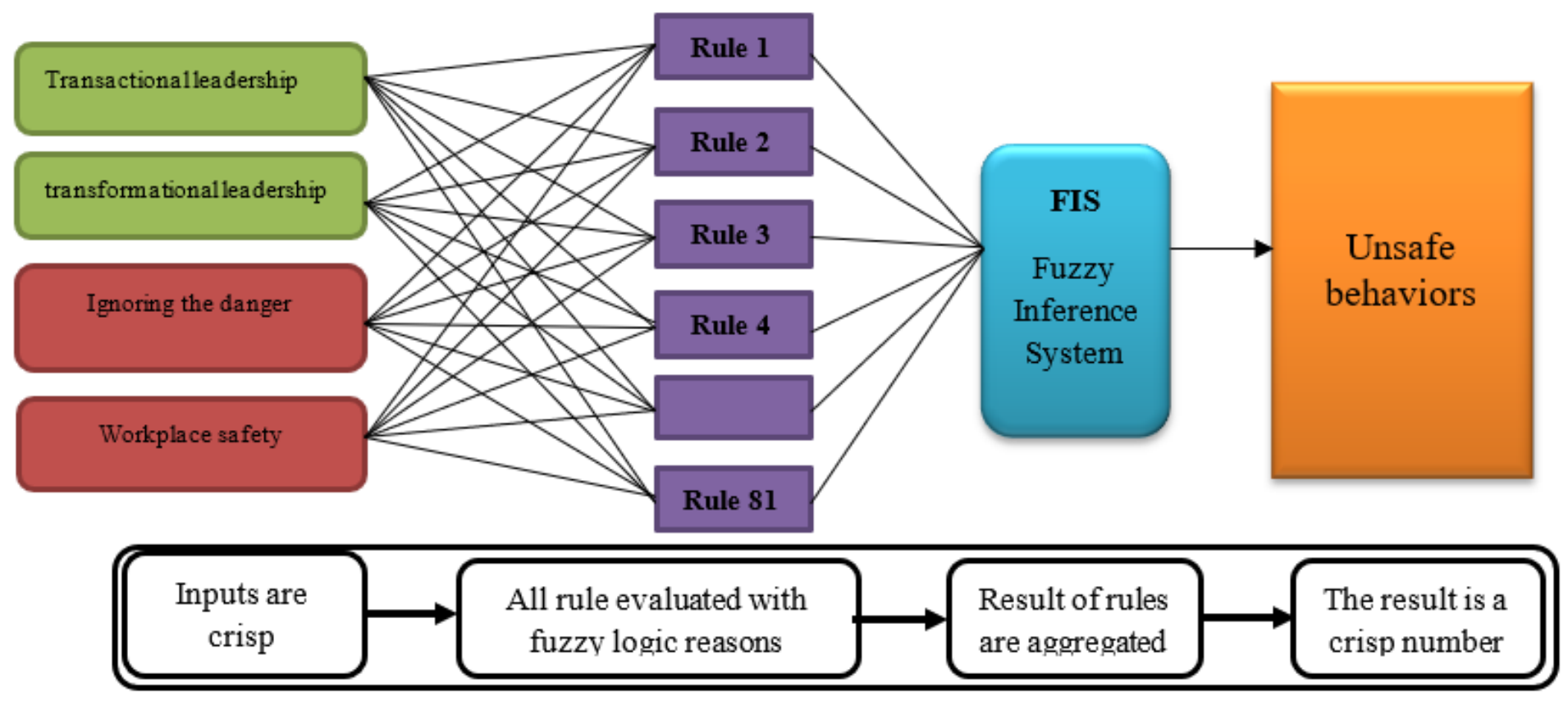

Figure 1

Schematic view of the performance of the fuzzy logic system

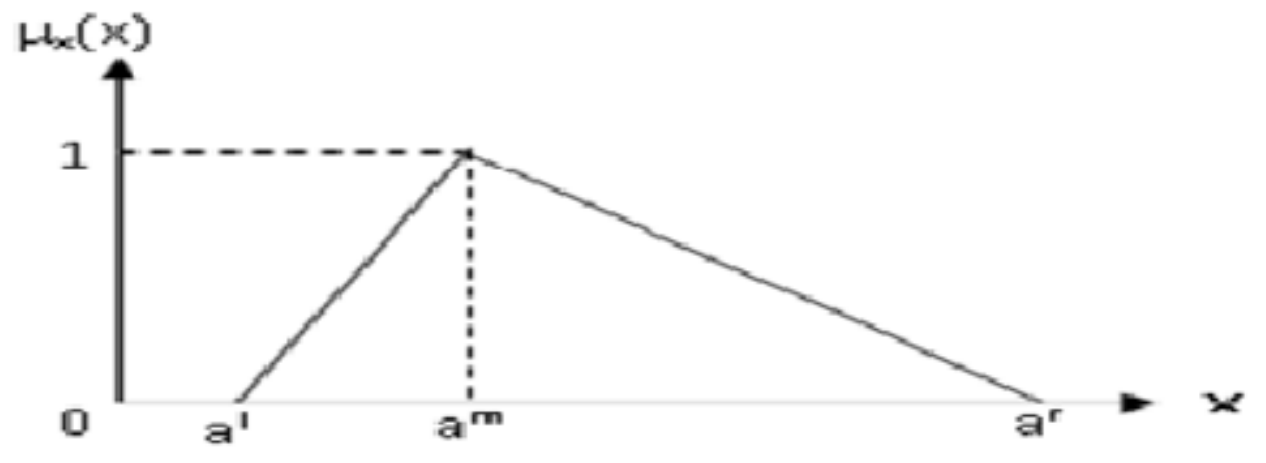

Figure 2

Triangular fuzzy number diagram 


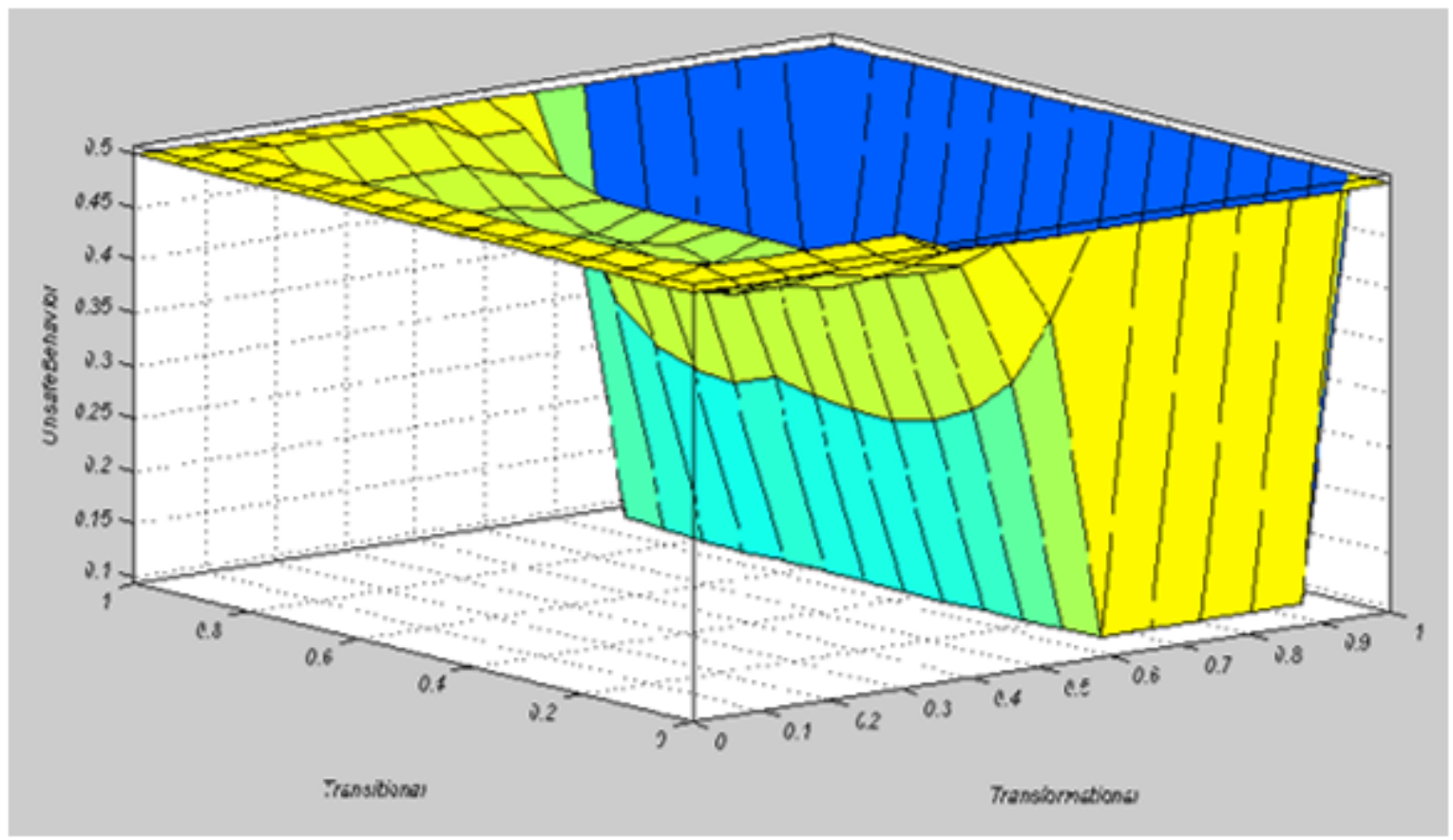

Figure 3

Fuzzy information cropping, transformational-transactional leadership style, and their feedback on occurrence of unsafe behavior

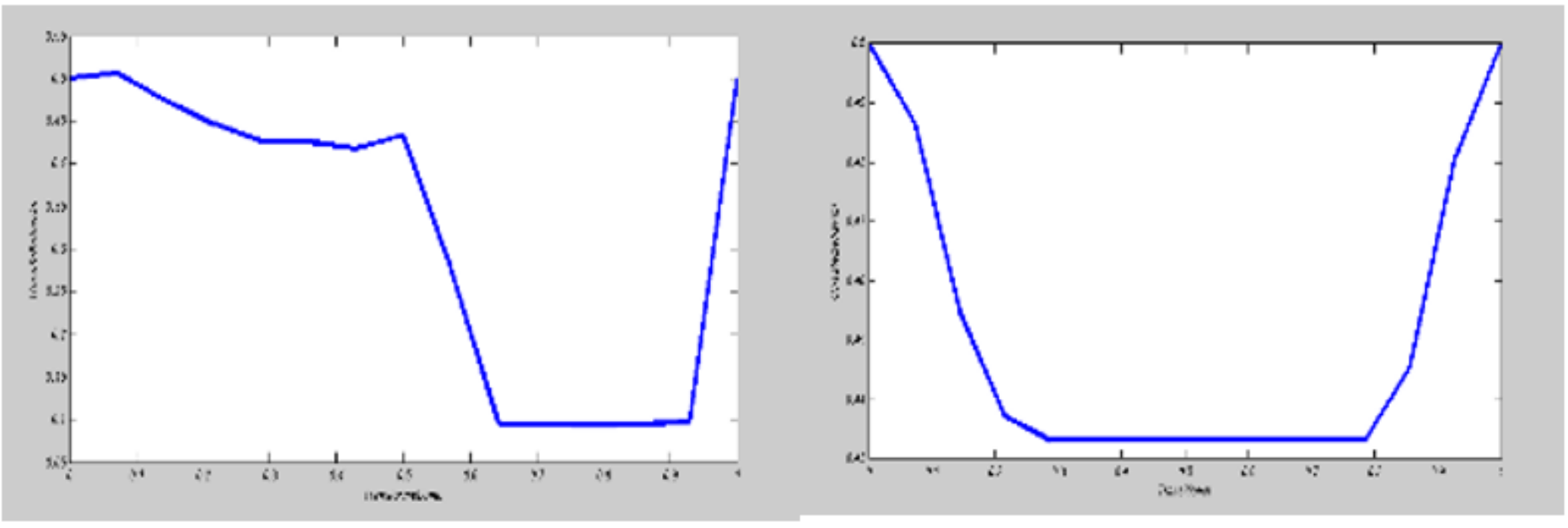

\section{Figure 4}

The relationship between leadership style and the occurrence of unsafe behaviors (Right: unsafe behavior - transactional leadership style / Left: unsafe behavior - transformational leadership style) 


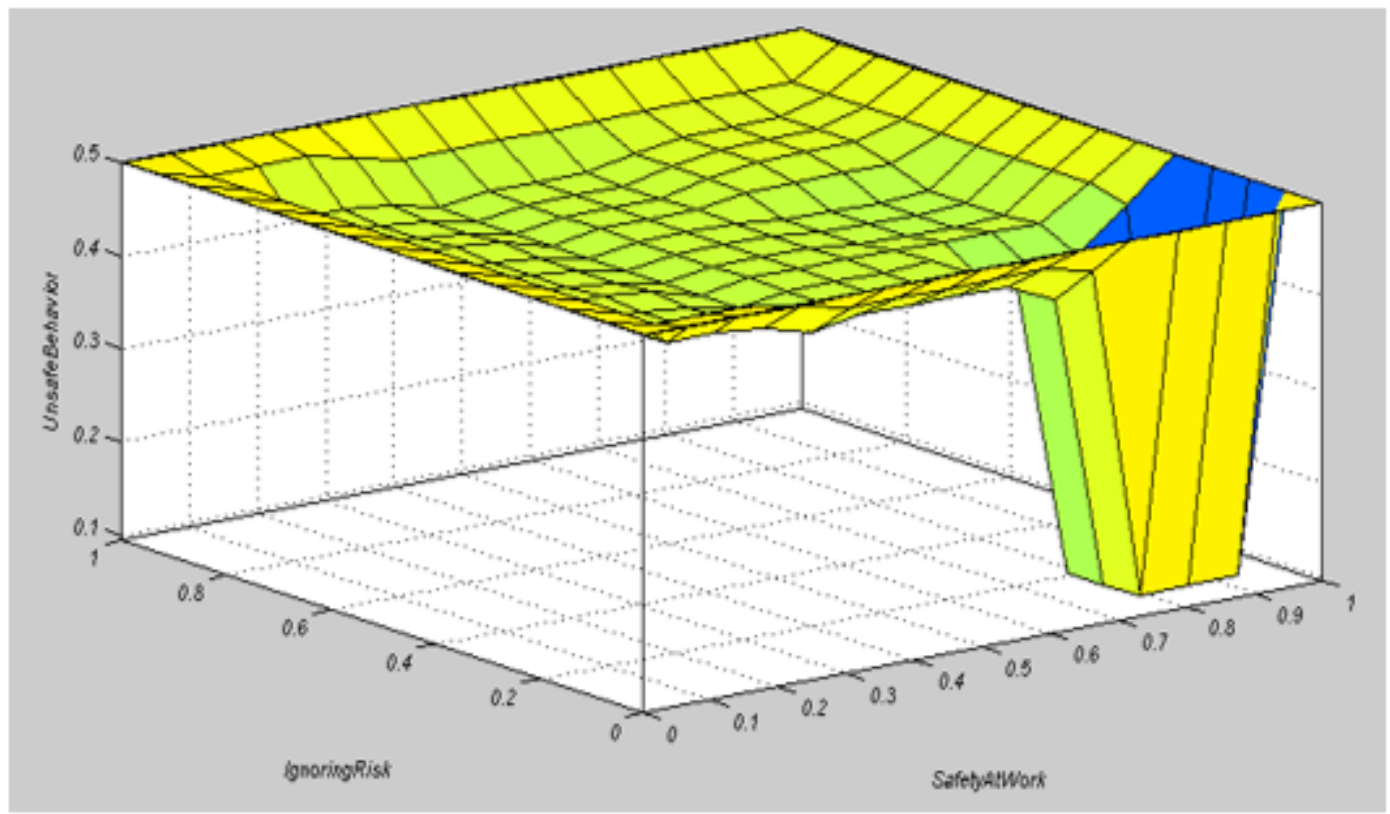

Figure 5

Fuzzy information cropping, dimensions of neglecting dangers and safety of workplace and their feedback on occurrence of unsafe behavior
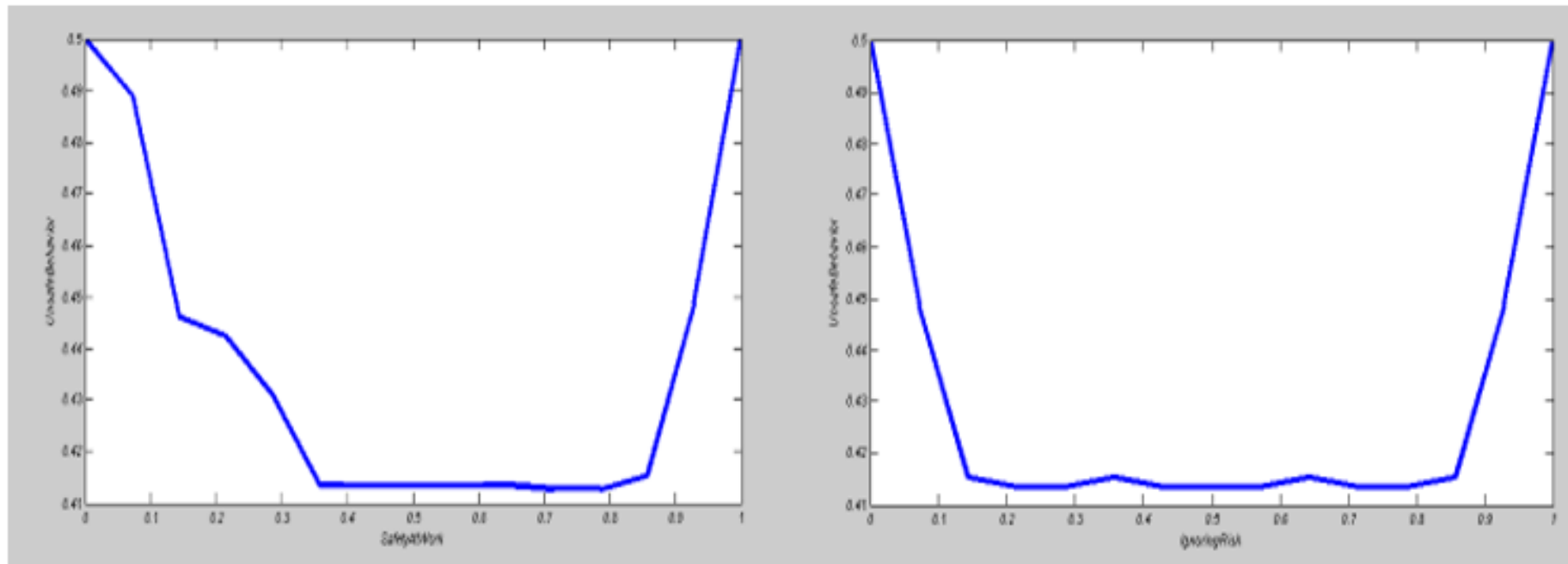

\section{Figure 6}

The relationship between safety climate dimensions and the occurrence of unsafe behaviors (Right: unsafe Behavior - neglecting dangers / Left: unsafe Behavior - safety of workplace) 